\title{
Magnetic resonance imaging-based measures predictive of short-term surgical outcome in patients with Chiari malformation Type I: a pilot study
}

\author{
Noam Alperin, PhD, ${ }^{1}$ James Ryan Loftus, BS, ${ }^{1}$ Ahmet M. Bagci, PhD, ${ }^{1}$ Sang H. Lee, MS, ${ }^{1}$ \\ Carlos J. Oliu, MD, ${ }^{1}$ Ashish H. Shah, MD, ${ }^{2}$ and Barth A. Green, MD² \\ Departments of ${ }^{1}$ Radiology and ${ }^{2}$ Neurological Surgery, University of Miami, Florida
}

OBJECTIVE This study identifies quantitative imaging-based measures in patients with Chiari malformation Type I (CM-I) that are associated with positive outcomes after suboccipital decompression with duraplasty.

METHODS Fifteen patients in whom CM-I was newly diagnosed underwent MRI preoperatively and 3 months postoperatively. More than 20 previously described morphological and physiological parameters were derived to assess quantitatively the impact of surgery. Postsurgical clinical outcomes were assessed in 2 ways, based on resolution of the patient's chief complaint and using a modified Chicago Chiari Outcome Scale (CCOS). Statistical analyses were performed to identify measures that were different between the unfavorable- and favorable-outcome cohorts. Multivariate analysis was used to identify the strongest predictors of outcome.

RESULTS The strongest physiological parameter predictive of outcome was the preoperative maximal cord displacement in the upper cervical region during the cardiac cycle, which was significantly larger in the favorable-outcome subcohorts for both outcome types $(p<0.05)$. Several hydrodynamic measures revealed significantly larger preoperative-topostoperative changes in the favorable-outcome subcohort. Predictor sets for the chief-complaint classification included the cord displacement, percent venous drainage through the jugular veins, and normalized cerebral blood flow with 93.3\% accuracy. Maximal cord displacement combined with intracranial volume change predicted outcome based on the modified CCOS classification with similar accuracy.

CONCLUSIONS Tested physiological measures were stronger predictors of outcome than the morphological measures in patients with CM-I. Maximal cord displacement and intracranial volume change during the cardiac cycle together with a measure that reflects the cerebral venous drainage pathway emerged as likely predictors of decompression outcome in patients with $\mathrm{CM}-\mathrm{I}$.

http://thejns.org/doi/abs/10.3171/2016.5.SPINE1621

KEY WORDS Chiari malformation; decompressive surgery outcome; CSF flow dynamics; MRI; multivariate analysis; congenital

0 UBOCCIPITAL decompressive surgery is the treatment of choice for Chiari malformation Type I (CM-I), yet unfavorable outcomes have been reported in approximately $30 \%$ of patients. ${ }^{1,17}$ In addition, it has been shown that in two-thirds of patients with an unfavorable outcome, adequate decompression is shown on MRI, suggesting that a lack of positive outcome is not a result of surgical failure but is likely a result of patient selection. ${ }^{21}$ Efforts have been made to identify imaging-based signs, subjective or quantitative, that are indicative of decompressive surgery outcome. Both morphological and hydrodynamic parameters have been investigated as potential predictors of outcome. Proposed parameters include small posterior fossa/supratentorial volume (STV) ratio $^{11}$ and absent or decreased biphasic CSF flow at the level of the foramen magnum ventral and dorsal to the cervical-medullary brainstem..$^{20,22}$ One study reported that peak CSF velocities below certain thresholds at the aqueduct and at the posterior C2-3 level are modest predictors of a lack of improvement after decompression. ${ }^{27}$ Other studies have reported that a preoperative-to-postoperative decrease in spatial heterogeneity of CSF flow, ${ }^{16}$ increased intracranial compliance (ICC), ${ }^{26}$ and increased posterior cranial fossa (PCF) volume by at least $15 \%{ }^{24}$ were associated with a good outcome.

An impediment to the identification of predictors of de-

ABBREVIATIONS CCOS = Chicago Chiari Outcome Scale; CM-I = Chiari malformation Type I; ICC = intracranial compliance; ICP = intracranial pressure; ICV = intracranial volume; ICVC = ICV change; MRI-ICP = MRI-derived ICP; PCF = posterior cranial fossa; STV = supratentorial volume.

SUBMITTED January 4, 2016. ACCEPTED May 26, 2016.

INCLUDE WHEN CITING Published online August 5, 2016; DOI: 10.3171/2016.5.SPINE1621. 
compression outcome is the lack of standardized reporting of surgical outcomes. Aiming to alleviate this problem, Aliaga et al. ${ }^{1}$ developed the Chicago Chiari Outcome Scale (CCOS), a disease-specific standardized outcome scale that was independently validated by Yarbrough et al. ${ }^{28}$ According to a recent meta-analysis, the CCOS was the only CM-I-specific outcome scale that correlated with well-accepted measures of outcome..$^{14}$

Our study follows a previous comparison between a larger cohort of patients with CM-I and healthy agematched controls that identified, in addition to tonsillar herniation, 10 imaging-based quantitative morphological and hydrodynamic markers of CM-I. ${ }^{6}$ In a subcohort of the patients with CM-I who underwent decompression, duraplasty, and lysis of arachnoid adhesions, postsurgical MRI and clinical evaluation were performed to help us identify imaging-based measures predictive of surgical outcome for improved management of CM-I. In addition, we also assessed which postsurgical changes were associated with either a positive or negative surgical outcome.

\section{Methods}

\section{Patients}

After approval by the University of Miami institutional review board, 15 patients (12 females, mean age $35 \pm$ 9 years) with radiologically defined CM-I who gave informed consent were included in this study. None of the patients had hydrocephalous, a syrinx was seen in only 3 of the 15 patients, 2 of the patients' conditions were considered to fit into the category of Chiari malformation Type $1.5,2$ patients had a retroflexed odontoid, and 1 patient had a Klippel-Feil anomaly at $\mathrm{C} 2-3$. Each patient underwent surgery performed by the same neurosurgeon at the same institution. Symptoms, surgical procedure, and clinical outcome were compiled from questionnaires completed by the patients before and after surgery, surgical reports, and office notes for preoperative and postoperative followup visits. Patient demographics and clinical presentations are listed in Table 1.

\section{Surgical Technique}

The basic suboccipital decompression performed in each patient included C-1 laminectomy and craniectomy (approximately $3 \times 3 \mathrm{~cm}$ ). Using an intraoperative microscope, intradural exploration was undertaken along with bilateral tonsillopexy via bipolar coagulation and lysis of arachnoid adhesions. An expansile duraplasty was closed with an autologous graft if sufficient graft could be harvested; otherwise, an allograft was used. Blood products were allowed to clear the subarachnoid space through a lumbar CSF drain to minimize scarring and inflammation. Patients underwent intraoperative neuromonitoring. A description of the surgical procedure performed for each patient is listed in Table 2.

\section{Surgical Outcome Reporting}

Two approaches were used for assessing surgical outcome. The first approach simply accounted for the chief complaint of each patient; each surgery was categorized as having either failed or succeeded based on worsening or no effect versus improvement or resolution of the chief complaint (e.g., suboccipital headache). The second approach used an outcome scale based on the CCOS., ${ }^{1,28}$ The CCOS is a 4-category scale with scores ranging from 1 to 4 (i.e., from worsened to resolved) in each of the following categories: pain symptoms (e.g., cough, headache), nonpain symptoms (e.g., dysphagia, paresthesias), functionality (ability to attend school/work), and surgical complications. ${ }^{1}$ Although score categories of 4-8, 9-12, and 13-16 were proposed originally for patients whose outcome was worse, unchanged, or improved from baseline, respectively, a validation study showed that a cutoff

TABLE 1. Demographics and clinical presentation

\begin{tabular}{|c|c|c|c|c|c|c|c|}
\hline $\begin{array}{l}\text { Case } \\
\text { No. }\end{array}$ & $\begin{array}{c}\text { Age (yrs) } \\
\text { Sex }\end{array}$ & $\begin{array}{l}\text { CM } \\
\text { Type }\end{array}$ & Syrinx & $\begin{array}{l}\text { Retroflexed } \\
\text { Odontoid }\end{array}$ & CCJ Anomaly & $\begin{array}{l}\text { Steroids } \\
\text { Used }\end{array}$ & Complications \\
\hline 1 & $49, \mathrm{~F}$ & 1.5 & No & No & No & No & None \\
\hline 2 & $21, \mathrm{~F}$ & 1 & No & Yes & Klippel-Feil C-2/C-3 & No & Pseudomeningocele (blood patch) \\
\hline 3 & $36, \mathrm{~F}$ & 1 & No & No & No & No & Pseudomeningocele (surgery) \\
\hline 4 & $48, \mathrm{~F}$ & 1 & $\mathrm{C} 7-\mathrm{T} 2$ & No & No & No & None \\
\hline 5 & $45, M$ & 1 & C-2 (small) & No & No & No & None \\
\hline 6 & $39, \mathrm{~F}$ & 1 & No & No & No & No & None \\
\hline 7 & $32, \mathrm{~F}$ & 1 & No & No & No & No & None \\
\hline 8 & $27, \mathrm{M}$ & 1 & Thoracic & No & No & No & None \\
\hline 9 & $22, \mathrm{~F}$ & 1 & No & Yes & No & No & None \\
\hline 10 & $36, F$ & 1 & No & No & No & Yes & None \\
\hline 11 & $23, \mathrm{M}$ & 1 & No & No & No & No & None \\
\hline 12 & $36, F$ & 1.5 & No & No & No & No & Infection \\
\hline 13 & $29, \mathrm{~F}$ & 1 & No & No & No & No & None \\
\hline 14 & $29, \mathrm{~F}$ & 1 & No & No & No & No & None \\
\hline 15 & $45, F$ & 1 & Presyrinx, cervical & No & No & No & None \\
\hline
\end{tabular}

$\mathrm{CCJ}=$ craniocervical junction . 


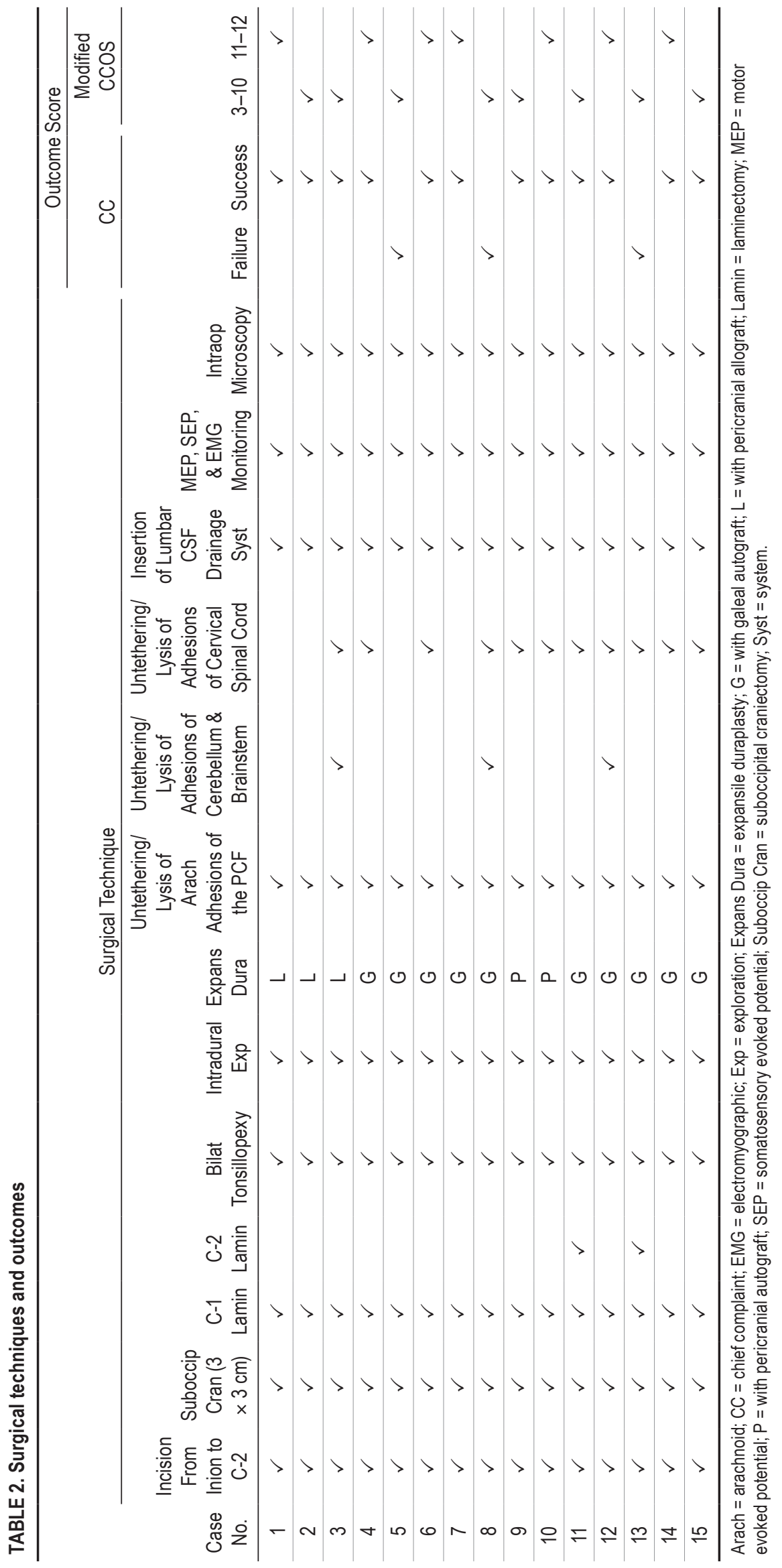


score of 14 had the best receiver operating characteristic curve for identifying an "improved" outcome. ${ }^{28}$ The same study also showed that the functionality subscore, an especially subjective and difficult assessment, had no impact on the ability of the CCOS to successfully differentiate outcomes, and a score based on the other 3 categories had similarly good sensitivity and specificity for identifying patients whose condition had improved. Our study made use of these findings by taking into account pain symptoms, nonpain symptoms, and complications with a cutoff of 11 and above for patients whose condition had improved, consistent with the validation study's cutoff of 14 .

\section{MRI and Data Acquisition}

MRI scans were performed using a 3-T scanner (Magnetom Trio, Siemens Healthcare). The imaging protocol included 3D T1-weighted (magnetization-prepared rapid gradient-echo [MPRAGE]) and T2-weighted (sampling perfection with application-optimized contrasts using different flip-angle evolutions [SPACE]) sequences with $1-\mathrm{mm}$ isotropic resolution for quantitative morphological assessments. Velocity-encoding images of blood and CSF flows were obtained by using high $(70-80 \mathrm{~cm} / \mathrm{sec})$ and low $(7-8 \mathrm{~cm} / \mathrm{sec})$ velocity-encoded cine phase-contrast scans. The voxels had a pixel size of $0.56 \times 0.56 \mathrm{~mm}$ and a slice thickness of $5 \mathrm{~mm}$. Preoperative scans were acquired an average of $6.8 \pm 13.2$ weeks before surgery, and postoperative scans were obtained an average of $12.9 \pm 2.2$ weeks after the decompressive procedure. The morphological and physiological parameters extracted from the MRI scans included linear and volumetric morphological measures of the PCF, CSF flow and tissue motion, and intracranial hydrodynamics measures derived from measurements of the blood and CSF flow to and from the cranium. The extracted quantitative measures are listed in Table 3.

\section{Linear and Volumetric Measurements}

Linear measures were acquired by an experienced observer, blinded to the surgical outcome, using an in-house image-display tool. The assessed 1D measures included the clivus, supraocciput, Twining's line, McRae's line, maximum perpendicular distance between basion and posterior inferior portion of $\mathrm{C}-2$, and clivoaxial angle, which were measured on midsagittal T1-weighted images. Posterior cranial fossa volumetry was performed by using a previously described automated method. ${ }^{12}$ With this method, the PCF, hindbrain, and fourth ventricle volumes were quantified using automated segmentation guided by a CM-I-specific brain atlas. These data provide a measure of hindbrain crowdedness as the ratio of hindbrain/PCF volume. The PCF volume/STV ratio was calculated for comparison with a previous work that incorporated this parameter. ${ }^{11}$ The STV was obtained by subtracting the PCF volume from the intracranial volume (ICV).

\section{CSF Flow and Cord Motion}

Details concerning the methods for deriving CSF flow and cord motion along with the intracranial hydrodynamics measures (ICV change [ICVC], ICC index, and intracranial pressure [ICP]) have been described in past studies. ${ }^{6,79}$ In brief, blood and CSF volumetric flow rates into and out from the cranium are obtained using the pulsatility-based segmentation method for the measurement of pulsatile flow. ${ }^{2}$ This technique uses dynamic information throughout the cardiac cycle along with a cross-correlation method to differentiate lumen from background pixels and delineate the lumen boundary. ${ }^{2}$ Volumetric flow rates are obtained by integrating the velocities with all the pixels within the delineated lumen. The mean velocity in each phase of the cardiac cycle is the average velocity within the lumen boundary. The craniospinal CSF stroke volume, the CSF volume that moves back and forth between the cranial and spinal compartments during 1 cardiac cycle, is obtained by time integration of the volumetric flow-rate waveforms. The cord-displacement waveform during the cardiac cycle is obtained by time integration of the average velocity waveform in a region of interest covering the center of the cord. Potential errors caused by baseline drift are corrected by using a constraint that renders the net displacement over the entire cardiac cycle to zero.

\section{Intracranial Hydrodynamics Measures}

Derivation of ICP by MR has been described ${ }^{9}$ and externally validated..$^{23}$ The ICP is derived from ICC by using

TABLE 3. Quantitative MRI-derived morphological and physiological measures

\begin{tabular}{|c|c|c|c|c|}
\hline Linear Morphology & 3D Morphology & CSF Flow \& Cord Motion & CBF & Intracranial Hydrodynamics \\
\hline Tonsillar herniation & 4th ventricle vol & CSF stroke vol & TCBF & ICVC \\
\hline Clivus & ICV & Systolic CSF velocity & $\begin{array}{l}\% \text { venous drainage } \\
\text { through jugular veins }\end{array}$ & Pulse pressure gradient \\
\hline Supraocciput & PCFV & $\begin{array}{l}\text { Systolic CSF velocity inho- } \\
\text { mogeneity (SD) }\end{array}$ & $\begin{array}{l}\text { CBF normalized for brain } \\
\text { tissue vol }\end{array}$ & ICC index \\
\hline Twining's line & Hindbrain vol & Diastolic CSF velocity & & MRI-ICP \\
\hline McRae's line & PCFV/ICV & $\begin{array}{l}\text { Diastolic CSF velocity inho- } \\
\text { mogeneity (SD) }\end{array}$ & & \\
\hline $\mathrm{pBC2}$ & Hindbrain crowdedness (HBV/PCFV) & Maximum cord displacement & & \\
\hline Clivoaxial angle & PCFVISTV & & & \\
\hline
\end{tabular}

$\mathrm{CBF}=$ cerebral blood flow; $\mathrm{HBV}=$ hindbrain volume; $\mathrm{pBC2}=$ maximum perpendicular distance between basion and posterior inferior portion of $\mathrm{C}-2 ; \mathrm{PCFV}=\mathrm{PCF}$ volume; $\mathrm{SD}=$ standard deviation; $\mathrm{TCBF}=$ total $\mathrm{CBF}$. 
the monoexponential relationship between pressure and volume. Compliance is derived from the ratio of the ICV and ICP changes that occur with each cardiac cycle. The ICVC during the cardiac cycle is obtained from the momentary differences between total cerebral blood inflow, venous blood outflow, and craniospinal CSF that enter and leave the cranium during a cardiac cycle. Maximal change in pressure is derived from changes in the CSF pressure gradient calculated using the Navier-Stokes relationship between pressure gradients and CSF velocities. ${ }^{9,19}$ Total cerebral blood inflow is calculated by the summation of flow through the internal carotid and vertebral arteries, and venous outflow is calculated by the summation of the flow through the internal jugular veins and secondary veins (e.g., epidural and vertebral) if present.

\section{Statistical Analysis}

Statistical analyses included comparisons of preoperative and preoperative-to-postoperative changes in patient measures within the 2 surgical outcome scales used in this study. An unpaired 2-tailed Student t-test for unequal variances was performed within the outcome scales between subcohorts using Microsoft Excel; $p<0.05$ was considered significant. In addition, multivariate analysis was used to construct sets of variables predictive of surgical outcome using SPSS software. First, bivariate logistic regression was performed with each measured parameter individually to assess its significance for each outcome scale. Those with a significance of $<0.2$ were entered through a backward likelihood-ratio logistic regression to identify a predictor set. If a pair happened to be significantly correlated, the less significant of the 2 variables was removed from the test. The predictor set was then run through a discriminant analysis to determine predictive value in placement within the outcome scale for which it was created.

\section{Results}

Predecompression and postdecompression MRIs from one of the patients demonstrating surgical anatomic outcome are shown in Fig. 1. Three of the 15 patients had their chief complaint unchanged or worsened, whereas 12 had their chief complaint resolved or improved. Using the modified CCOS, 8 patients scored 10 or lower, indicating a worsened or unchanged outcome (grouped as treatment failure), and the remaining 7 patients scored 11-12 (good outcome). It is notable that all patients whose chief complaint did not improve were also in the CCOS unfavorable-outcome group. Of the 15 patients, 12 had no complications, 2 had transient complications (pseudomeningocele and infection), and 1 had persistent pseudomeningocele that required multiple lumbar punctures to drain CSF. Only 1 of the 3 patients with complications, the patient with a large cervical syrinx, had a favorable outcome according to both classifications.

Using the chief-complaint approach for outcome classification, the only statistically significant difference in preoperative measurements between the unimproved and improved cohorts was cord displacement (mean \pm SD $183 \pm$ 12 vs $278 \pm 126 \mu \mathrm{m}$, respectively; $\mathrm{p}=0.02$ ). The physiological measure of percent cerebral venous drainage through the jugular veins trended toward being smaller in the fail-
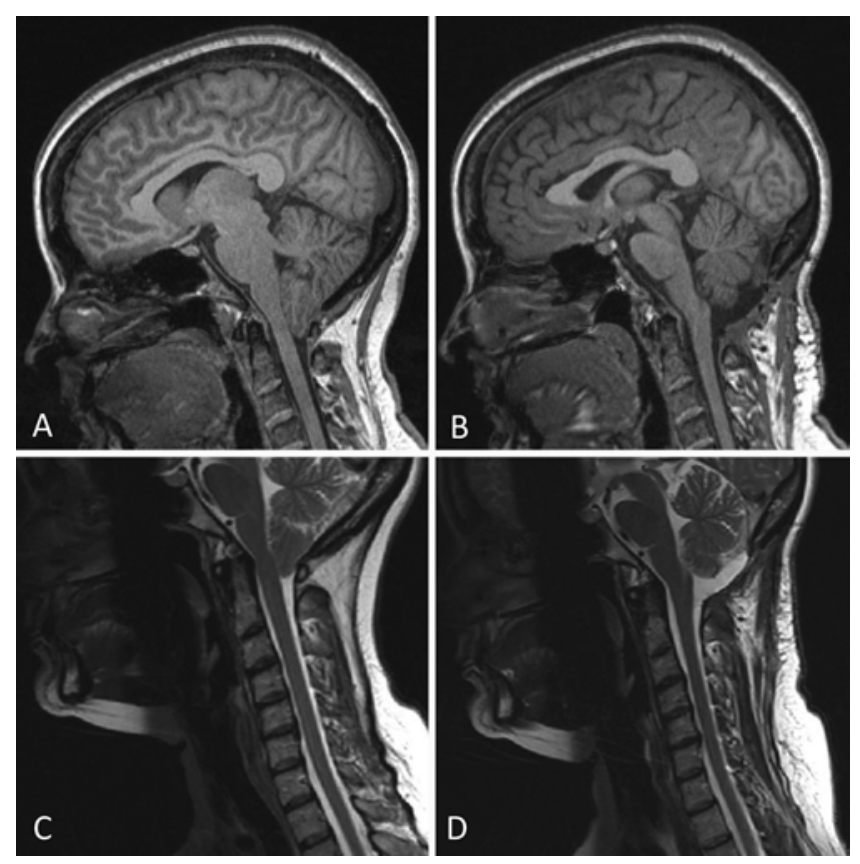

FIG. 1. Sagittal T1-weighted ( $A$ and $B$ ) and T2-weighted ( $C$ and $D$ ) images of a patient with $C M$-l before ( $A$ and $C$ ) and after ( $B$ and $D)$ surgery. Postoperative MRI demonstrated the increase in CSF volume in the PCF.

ure subcohort than in the success subcohort (mean $41.8 \pm$ 40.3 vs $70.8 \pm 13.6 \%$, respectively; $p=0.34$ ). The preoperative morphological measures, including PCF crowdedness, PCF volume/STV ratio, and tonsillar descent, all within the previously determined CM-I range, ${ }^{6}$ were not statistically different between the unfavorable- and favorable-outcome cohorts. Mean preoperative MRI measures for the 2 sets of these 2 surgical outcome cohorts are listed in Table 4.

Two preoperative physiological measures were significantly different between the CCOS-based unfavorableand favorable-outcome groups, cord displacement (mean $199 \pm 92$ vs $329 \pm 111 \mu \mathrm{m}$, respectively; $\mathrm{p}=0.03$ ) and ICVC (mean $0.60 \pm 0.18$ vs $0.37 \pm 0.11 \mathrm{ml}$, respectively; $\mathrm{p}=0.01$ ). Patients who had large cord displacement and small ICVC before surgery tended to benefit more from the operation. As with the chief-complaint outcome characterization, no significant difference in the morphological measures were observed between the 2 outcome cohorts. Significant preoperative-to-postoperative differences in hydrodynamic measures were seen between the improved and unimproved cohorts. Using chief complaint for outcome classification, a significantly greater mean increase in ICVC was seen in patients with a favorable outcome $(-0.05 \pm$ $0.02 \mathrm{ml}$ [unimproved cohort] vs $0.12 \pm 0.22 \mathrm{ml}$ [improved cohort]; $\mathrm{p}=0.02)$, which is consistent with similar trends seen with MRI-derived ICP (MRI-ICP) and ICC index. In the patients with a favorable outcome, the mean ICC index increased more $(-1.12 \pm 1.76$ [unimproved cohort] vs 2.75 \pm 5.05 [improved cohort], respectively; $p=0.053$ ) and pressure (MRI-ICP), which is inversely related, was reduced more (mean $0.77 \pm 2.06 \mathrm{~mm} \mathrm{Hg}$ [unimproved cohort] vs $-2.83 \pm 4.87 \mathrm{~mm} \mathrm{Hg}$ [improved cohort], respectively; $\mathrm{p}=$ $0.08)$ than in those with an unfavorable outcome. In ad- 
TABLE 4. Mean preoperative MRI parameters in poor and good surgical outcomes

\begin{tabular}{|c|c|c|c|c|c|c|}
\hline \multirow[b]{2}{*}{ Parameter } & \multicolumn{2}{|c|}{$\mathrm{CC}(\mathrm{n}=15)$} & \multirow[b]{2}{*}{ p Value } & \multicolumn{2}{|c|}{ Modified CCOS Score $(n=15)$} & \multirow[b]{2}{*}{ p Value } \\
\hline & Failure $(n=3)$ & Success $(n=12)$ & & $3-10(n=8)$ & $11-12(n=7)$ & \\
\hline \multicolumn{7}{|l|}{ 1D morphology } \\
\hline Tonsillar herniation (mm) & $7.2 \pm 5.1$ & $8.5 \pm 3.0$ & 0.70 & $7.6 \pm 4.1$ & $9.0 \pm 2.3$ & 0.42 \\
\hline Length of clivus (mm) & $38.3 \pm 4.5$ & $36.5 \pm 3.3$ & 0.56 & $37.0 \pm 3.5$ & $36.7 \pm 3.8$ & 0.88 \\
\hline Length of supraocciput (mm) & $37.3 \pm 4.7$ & $37.9 \pm 3.9$ & 0.86 & $38.1 \pm 3.4$ & $37.4 \pm 4.6$ & 0.75 \\
\hline $\mathrm{pBC2}(\mathrm{mm})$ & $7.8 \pm 2.0$ & $7.0 \pm 1.6$ & 0.58 & $7.7 \pm 2.0$ & $6.6 \pm 0.9$ & 0.17 \\
\hline $\operatorname{CXA}\left({ }^{\circ}\right)$ & $141.3 \pm 12.5$ & $141.8 \pm 6.1$ & 0.95 & $141.6 \pm 9.3$ & $141.9 \pm 4.5$ & 0.95 \\
\hline \multicolumn{7}{|l|}{ 3D morphology } \\
\hline Fourth ventricle vol (ml) & $1.22 \pm 0.01$ & $1.27 \pm 0.40$ & 0.68 & $1.31 \pm 0.39$ & $1.21 \pm 0.33$ & 0.58 \\
\hline PCFV (ml) & $181 \pm 9$ & $182 \pm 15$ & 0.82 & $178 \pm 14$ & $187 \pm 13$ & 0.20 \\
\hline ICV (ml) & $1358 \pm 82$ & $1326 \pm 142$ & 0.64 & $1311 \pm 136$ & $1357 \pm 130$ & 0.52 \\
\hline PCFV/ICV & $0.133 \pm 0.007$ & $0.138 \pm 0.008$ & 0.40 & $0.136 \pm 0.010$ & $0.138 \pm 0.006$ & 0.60 \\
\hline Hindbrain crowdedness (HBV/PCFV) & $0.890 \pm 0.018$ & $0.893 \pm 0.020$ & 0.86 & $0.890 \pm 0.022$ & $0.895 \pm 0.018$ & 0.68 \\
\hline PCFVISTV & $0.153 \pm 0.010$ & $0.160 \pm 0.011$ & 0.39 & $0.158 \pm 0.013$ & $0.160 \pm 0.008$ & 0.61 \\
\hline \multicolumn{7}{|l|}{ CSF flow \& tissue motion } \\
\hline CSF stroke vol (ml) & $0.49 \pm 0.23$ & $0.52 \pm 0.19$ & 0.86 & $0.47 \pm 0.19$ & $0.57 \pm 0.19$ & 0.34 \\
\hline CSF systolic velocity (mm/sec) & $18.9 \pm 3.6$ & $19.4 \pm 8.3$ & 0.87 & $18.8 \pm 7.1$ & $19.9 \pm 8.5$ & 0.79 \\
\hline $\begin{array}{l}\text { CSF systolic velocity inhomogeneity } \\
\text { (SD) }\end{array}$ & $8.2 \pm 1.8$ & $9.4 \pm 3.2$ & 0.42 & $8.7 \pm 2.0$ & $9.8 \pm 3.9$ & 0.54 \\
\hline CSF diastolic velocity (mm/sec) & $-9.6 \pm 0.4$ & $-11.0 \pm 2.8$ & 0.13 & $-9.8 \pm 2.4$ & $-11.8 \pm 2.4$ & 0.12 \\
\hline $\begin{array}{l}\text { CSF diastolic velocity inhomogeneity } \\
\text { (SD) }\end{array}$ & $6.8 \pm 1.5$ & $7.8 \pm 1.8$ & 0.41 & $7.1 \pm 1.3$ & $8.1 \pm 2.1$ & 0.27 \\
\hline Maximal cord displacement $(\mu \mathrm{m})$ & $183 \pm 12$ & $278 \pm 126$ & $0.02^{*}$ & $199 \pm 92$ & $329 \pm 111$ & $0.03^{*}$ \\
\hline \multicolumn{7}{|l|}{ Cerebral blood flow } \\
\hline TCBF (ml/min) & $725 \pm 82$ & $792 \pm 100$ & 0.30 & $735 \pm 96$ & $829 \pm 78$ & 0.06 \\
\hline $\mathrm{nCBF}(\mathrm{ml} / \mathrm{min}$ per $100 \mathrm{~g})$ & $83.9 \pm 6.2$ & $94.4 \pm 10.4$ & 0.07 & $88.9 \pm 8.7$ & $96.3 \pm 11.7$ & 0.20 \\
\hline Jugular drainage (\%) & $41.8 \pm 40.3$ & $70.8 \pm 13.6$ & 0.34 & $61.7 \pm 29.3$ & $68.8 \pm 13.5$ & 0.55 \\
\hline \multicolumn{7}{|l|}{ Intracranial hydrodynamics } \\
\hline PTP-PG (mm Hg/cm) & $0.037 \pm 0.004$ & $0.039 \pm 0.016$ & 0.73 & $0.039 \pm 0.014$ & $0.038 \pm 0.016$ & 0.90 \\
\hline ICVC (ml) & $0.56 \pm 0.26$ & $0.48 \pm 0.18$ & 0.64 & $0.60 \pm 0.18$ & $0.37 \pm 0.11$ & $0.01^{*}$ \\
\hline ICC index & $10.47 \pm 9.12$ & $8.67 \pm 4.03$ & 0.77 & $10.93 \pm 5.39$ & $6.85 \pm 3.82$ & 0.11 \\
\hline MRI-ICP (mm Hg) & $10.03 \pm 5.67$ & $10.04 \pm 4.86$ & 0.99 & $8.06 \pm 3.82$ & $12.30 \pm 5.09$ & 0.10 \\
\hline
\end{tabular}

$\mathrm{CXA}=$ clivoaxial angle; HBV = hindbrain volume; $\mathrm{nCBF}$ = cerebral blood flow normalized for brain tissue volume; PTP-PG = peak-to-peak pressure gradient .

* Statistically significant result.

dition, cord displacement showed a lower decrease in the unfavorable-outcome group than in the favorable-outcome group (mean $-10 \pm 26$ vs $-102 \pm 118 \mu \mathrm{m}$, respectively; $\mathrm{p}$ $=0.03$ ). A complete list of the magnitude of preoperativeto-postoperative change in measures sorted according to outcome is in Table 5.

When the hydrodynamic parameters in the CCOSbased groupings were examined, the failed- and successful-treatment subgroups essentially paralleled the chiefcomplaint-based groupings, and there were significant preoperative-to-postoperative differences in ICVC (mean $-0.05 \pm 0.07 \mathrm{ml}$ [unimproved cohort] vs $0.24 \pm 0.21 \mathrm{ml}$ [improved cohort]; $\mathrm{p}=0.01$ ) and MRI-ICP (mean $0.39 \pm$ $2.31 \mathrm{~mm} \mathrm{Hg}$ [unimproved cohort] vs $-4.96 \pm 5.11 \mathrm{~mm} \mathrm{Hg}$ [improved cohort]; $\mathrm{p}=0.03$ ), with a similar trend in ICC index (mean $0.12 \pm 4.92$ vs $4.11 \pm 3.96$; $p=0.11$ ). Corddisplacement changes followed the same trend as the chief-complaint outcomes, but this result was not significant (mean $-52 \pm 83 \mu \mathrm{m}$ [unimproved cohort] vs $-120 \pm$ $134 \mu \mathrm{m}$ [improved cohort]; $\mathrm{p}=0.27$ ). Examples of preoperative and postoperative net arterial minus venous blood flow and craniospinal CSF flow waveforms from a patient with an unfavorable outcome and a patient with a favorable outcome, demonstrating the respective postoperative decrease and increase in ICCs, are shown in Fig. 2. The relationship between the CSF and the blood flow waveforms shows the ICC state; the closer the CSF waveform follows the arterial-minus-venous waveform, the lesser the ICC. An example of preoperative and postoperative measurements of cord displacement from a patient with an unfavorable outcome and a patient with a favorable outcome, showing the larger postoperative reduction in cord displacement with the favorable-outcome group, are shown in Fig. 3. 
TABLE 5. Mean preoperative-to-postoperative changes in imaging parameters in different surgical outcomes

\begin{tabular}{|c|c|c|c|c|c|c|}
\hline \multirow[b]{2}{*}{ Parameter } & \multicolumn{2}{|c|}{$C C(n=15)$} & \multirow[b]{2}{*}{$\mathrm{p}$ Value } & \multicolumn{2}{|c|}{ Modified CCOS Score $(n=15)$} & \multirow[b]{2}{*}{$p$ Value } \\
\hline & Failure $(n=3)$ & Success $(n=12)$ & & $3-10(n=8)$ & $11-12(n=7)$ & \\
\hline \multicolumn{7}{|l|}{ 3D morphology } \\
\hline Fourth ventricle vol (ml) & $0.98 \pm 1.06$ & $0.39 \pm 0.39$ & 0.44 & $0.75 \pm 0.65$ & $0.23 \pm 0.37$ & 0.08 \\
\hline \multicolumn{7}{|l|}{ CSF flow \& tissue motion } \\
\hline CSF stroke vol (ml) & $-0.08 \pm 0.17$ & $-0.02 \pm 0.10$ & 0.57 & $-0.04 \pm 0.13$ & $-0.01 \pm 0.10$ & 0.63 \\
\hline CSF systolic velocity (mm/sec) & $-1.0 \pm 2.6$ & $0.4 \pm 4.1$ & 0.50 & $0.5 \pm 4.4$ & $-0.3 \pm 3.2$ & 0.67 \\
\hline CSF systolic velocity (SD) & $-0.2 \pm 1.5$ & $-0.3 \pm 3.6$ & 0.95 & $-0.2 \pm 1.2$ & $-0.3 \pm 4.7$ & 0.98 \\
\hline CSF diastolic velocity (mm/sec) & $-0.9 \pm 3.6$ & $-2.4 \pm 4.2$ & 0.58 & $-1.4 \pm 4.6$ & $-2.8 \pm 3.4$ & 0.51 \\
\hline CSF diastolic velocity (SD) & $0.1 \pm 1.2$ & $0.5 \pm 2.6$ & 0.74 & $0.3 \pm 1.9$ & $0.5 \pm 3.0$ & 0.87 \\
\hline Maximal cord displacement $(\mu \mathrm{m})$ & $-10 \pm 26$ & $-102 \pm 118$ & $0.03^{*}$ & $-52 \pm 83$ & $-120 \pm 134$ & 0.27 \\
\hline \multicolumn{7}{|l|}{ Cerebral blood flow } \\
\hline TCBF (ml/min) & $-14.7 \pm 123$ & $-32.2 \pm 99$ & 0.83 & $-32.9 \pm 77$ & $-22.7 \pm 132$ & 0.87 \\
\hline Jugular drainage (\%) & $-0.6 \pm 2.7$ & $-6.0 \pm 23.6$ & 0.46 & $-9.0 \pm 21.1$ & $-0.2 \pm 21.7$ & 0.44 \\
\hline \multicolumn{7}{|l|}{ Intracranial hydrodynamics } \\
\hline PTP-PG (mm Hg/cm) & $0.0003 \pm 0.006$ & $0.001 \pm 0.014$ & 0.84 & $0.001 \pm 0.014$ & $0.002 \pm 0.011$ & 0.82 \\
\hline ICVC (ml) & $-0.05 \pm 0.02$ & $0.12 \pm 0.22$ & $0.02^{*}$ & $-0.05 \pm 0.07$ & $0.24 \pm 0.21$ & $0.01^{*}$ \\
\hline ICC index & $-1.12 \pm 1.76$ & $2.75 \pm 5.05$ & $0.053 \dagger$ & $0.12 \pm 4.92$ & $4.11 \pm 3.96$ & 0.11 \\
\hline MRI-ICP (mm Hg) & $0.77 \pm 2.06$ & $-2.83 \pm 4.87$ & $0.08 \dagger$ & $0.39 \pm 2.31$ & $-4.96 \pm 5.11$ & $0.03^{*}$ \\
\hline
\end{tabular}

A multivariate analysis was performed to identify the preoperative measures that best discriminate between patients with a favorable outcome and those with an unfavorable outcome. In terms of the chief-complaint response, the following preoperative parameters were found to be the strongest predictors of outcome: maximal cord displacement, the percent venous drainage through the jugular veins, and normalized cerebral blood flow, which had an overall accuracy of $93.3 \%$, a sensitivity of $91.7 \%$, and a specificity of $100 \%$. The preoperative parameters that best predicted outcome classification based on the modified CCOS were maximal cord displacement and ICVC, which had 93.3\% accuracy, $85.7 \%$ sensitivity, and $100 \%$ specificity.

\section{Discussion}

Comparing imaging measures to clinical outcome revealed interesting new findings. Preoperative maximal cord displacement emerges as a strong indicator for surgical outcome. On average, patients with a large cord displacement had a favorable surgical outcome. This finding is consistent across both of the 2 outcome-classification approaches. The mean cord displacement of the patients who had a positive outcome in the modified-CCOS and the chief-complaint approaches were 329 and $278 \mu \mathrm{m}$, respectively, significantly above the mean values in the patients whose chief complaint did not improve (199 and 183 $\mu \mathrm{m}$, respectively) and well above the normative value of $174 \mu \mathrm{m}$, previously measured in control subjects. ${ }^{6}$ In fact, of the patients with a preoperative cord displacement of $\geq 300 \mu \mathrm{m}$, all had their chief complaint resolved or improved, and all but 1 patient scored 11-12 in the CCOSbased classification.
Spinal cord displacement was reported previously to be 1 of the 3 strongest imaging markers of CM-I. ${ }^{6}$ A large cord displacement is an indication of insufficient CSF venting from the cisterns into the spinal canal during the systolic phase, and therefore, brain tissue in the lower midbrain region is displaced to accommodate the increased systolic intracranial blood volume. It seems that effective decompressive surgery restores sufficient craniospinal CSF flow to accommodate the systolic increase in intracranial blood volume during each cardiac cycle. ${ }^{7,8}$

The measurement of cord displacement is derived from the transverse cine phase-contrast scan commonly performed to visualize and/or measure craniospinal CSF flow at the upper-cervical region. Therefore, this important indicator can be readily available as part of a routine clinical MRI scan for the diagnosis of CM-I.

Hofmann et al..$^{15}$ suggested that greater cord displacement is associated with greater obstruction of CSF flow at the foramen magnum, where the cord motion compensates for the obstructed CSF. The cord motion reflects the motion of the hindbrain during the cardiac cycle. During the systolic phase, mainly CSF is normally displaced into the spinal canal to accommodate the increased arterial inflow into the intracranial space. When craniospinal CSF outflow is restricted, or when the amount of CSF in the cisterna magna that can be displaced into the spinal canal is reduced as a result of a crowded PCF, a larger displacement of brain tissue occurs to accommodate the increased cerebral blood volume. Therefore, increased cord displacement is directly linked to the physiological alteration of the CSF pulsation that occurs in patients with CM-I.

The second preoperative measure indicative of outcome is the maximal change in ICV during the cardiac 

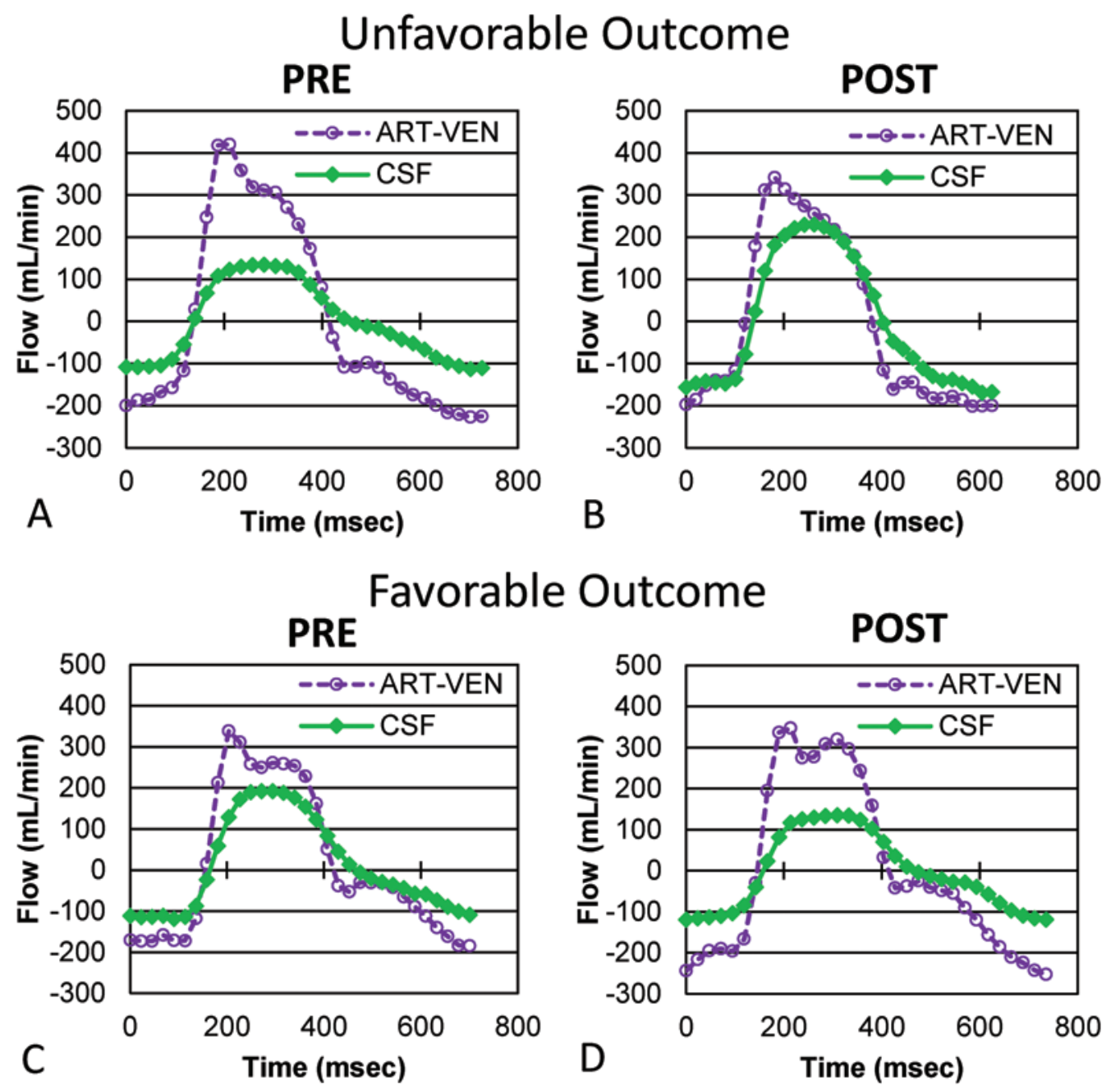

FIG. 2. Blood flow (arterial minus venous [ART-VEN]) and CSF flow waveforms from a patient with an unfavorable outcome (A and $B$ ) and a patient with a favorable outcome (C and D) obtained preoperatively and postoperatively. A postoperative decrease in compliance can be seen in panels $A$ and $B$ as the CSF waveform follows the ART-VEN waveform more closely postoperatively. The opposite, a postoperative increase in compliance, can be seen when comparing panels $C$ and $D$, in which the CSF waveform follows the ART-VEN waveform less closely postoperatively. Figure is available in color online only.

cycle (ICVC). A much lower mean ICVC $(0.37 \mathrm{ml})$ was measured in the patients with a favorable outcome (scoring 11-12 in the modified CCOS) than in the patients with an unfavorable outcome $(0.6 \mathrm{ml})$. A low ICVC reflects tightness of the intracranial space and its limited ability to accommodate the normal fluctuation in blood and CSF volumes that occur with every cardiac cycle. ${ }^{7}$ Furthermore, because ICC and MRI-ICP are both derivative of ICVC, both parameters demonstrated a trend for being indicative of outcome. The ICC index was lower (mean 6.85 vs 10.9, respectively; $\mathrm{p}=0.11$ ) and the MRI-ICP was higher (mean $12.3 \mathrm{vs} 8.1 \mathrm{~mm} \mathrm{Hg}$, respectively; $\mathrm{p}=0.10$ ) in the favorableoutcome cohort than in the unfavorable-outcome cohort. Yet, these differences did not reach statistical significance, likely because of the overall small number of subjects.

Unlike the physiological measures, the morphological measures, including those that are strong markers of CM-I, were not associated with outcome, because these measures were similar in the unfavorable- and favorable- outcome cohorts. The degrees of tonsillar herniation also were not different between the cohorts, which confirms the lack of specificity associated with this parameter. The similarity of values for this morphological measure among the 2 outcome cohorts is inconsistent with a previous report by Badie et al., ${ }^{11}$ who found that a small PCF/STV ratio was associated with a favorable outcome. These pilot data provide initial evidence for the importance of physiological measures for a comprehensive characterization of CM-I and treatment guidance.

The impact of surgery on the morphological and physiological measures was assessed by comparing the magnitude of the predecompression to postdecompression changes between the unfavorable- and favorable-outcome cohorts. It is interesting to note that the physiological measures related to the ICC demonstrated the strongest association with outcome. The patients with a favorable outcome had a significant preoperative-to-postoperative increase in ICVC compared with the unfavorable-outcome patients, 

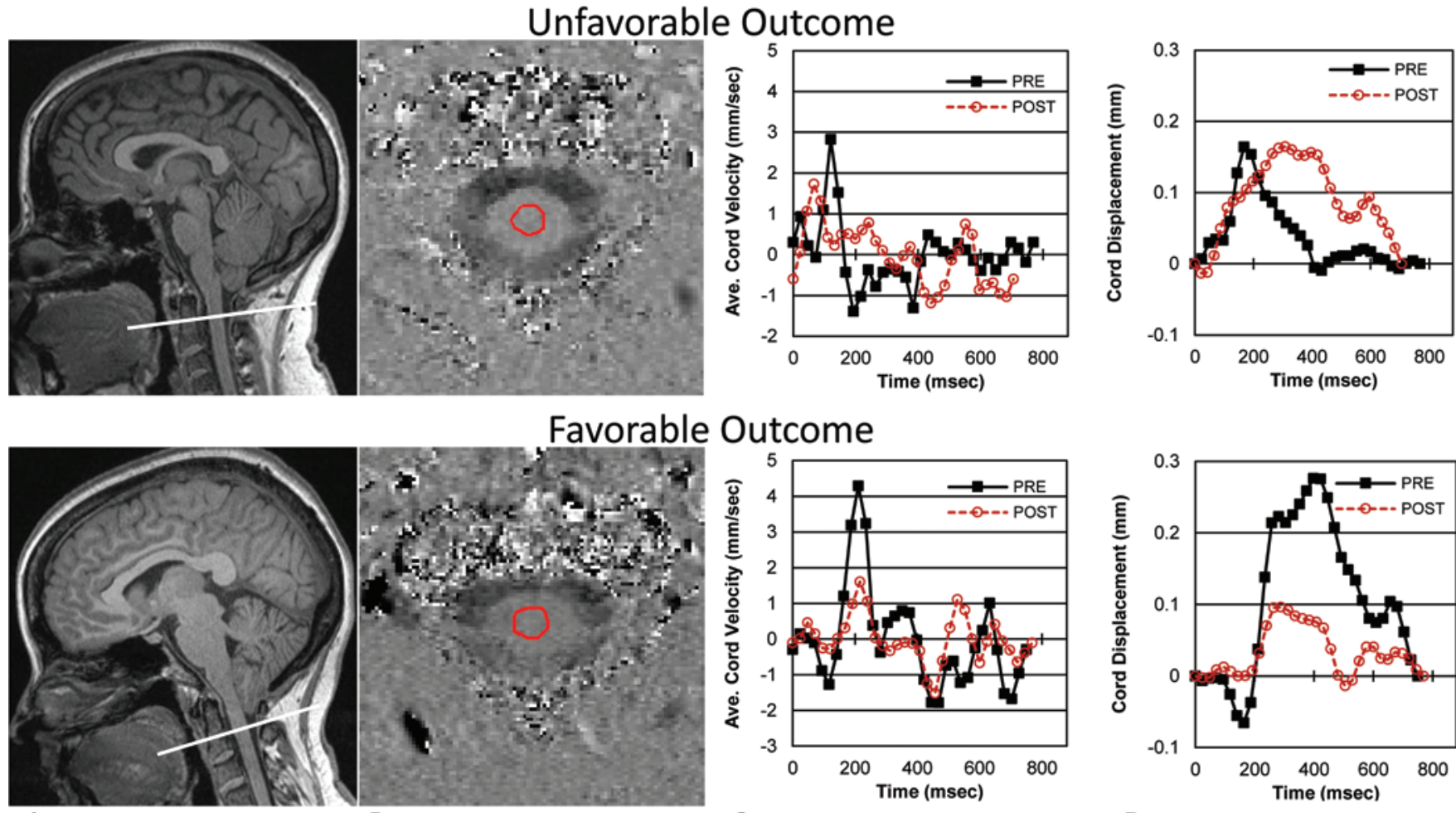

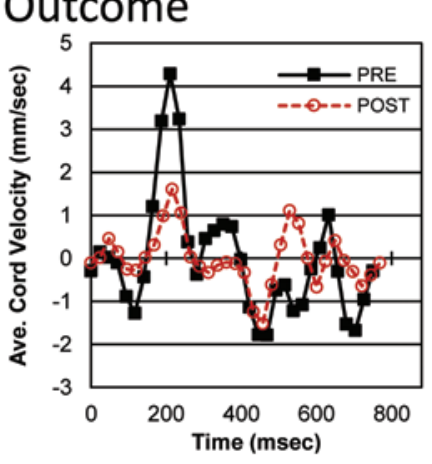

C

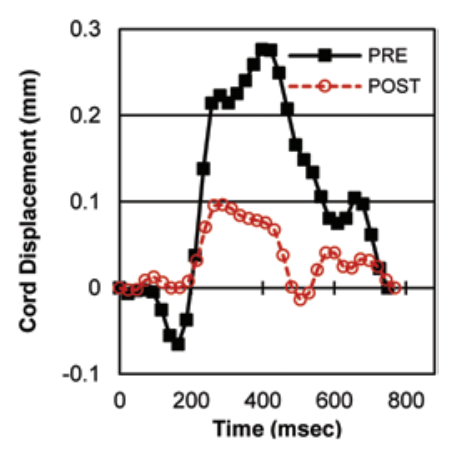

D

FIG. 3. Measurement of cord-motion dynamics obtained from a patient with an unfavorable outcome and a patient with a favorable outcome. A: Imaging plane superimposed on midsagittal MR images. B: Cord region of interest superimposed on phase-contrast MR images. C: Average cord-velocity waveforms for preoperative and postoperative scans. D: Cord-displacement waveforms from the preoperative and postoperative scans obtained by integration of the velocity waveforms with respect to time. Maximum cord displacements for preoperative and postoperative scans for the patient with an unfavorable outcome were 170 and $180 \mu \mathrm{m}$, respectively, and for the patient with a favorable outcome were 340 and $110 \mu \mathrm{m}$, respectively. Ave. = average. Figure is available in color online only.

in whom ICVC was nearly unchanged or even further decreased. ICVC is influenced by the volumetric reserves of the cranium and is used for determining the ICC, or the ability of the intracranial space to accommodate an increase of volume without a large increase in pressure. Therefore, the patients whose condition responded favorably to decompression demonstrated a significant increase in compliance and decrease in MRI-ICP (because ICP is inversely related to compliance). The current results are consistent with findings from a previous study of 12 patients who underwent decompressive surgery in which ICC index significantly increased in the patients who had a favorable outcome and decreased in a patient who reported poor outcome. ${ }^{26}$

In contrast with the hydrodynamic measures, the craniospinal CSF velocities and flow rates did not reveal a distinguishable pattern of change between the unfavorable- and favorable-outcome cohorts. This result is not consistent with findings in other studies that showed either increasing ${ }^{10,27}$ or decreasing ${ }^{13,16}$ peak velocities, depending on anatomical site, in positive responses to decompressive surgery. The lack of consistency related to CSF-velocity measures is likely a result of the documented large interindividual variability in CSF craniospinal velocities. ${ }^{8}$

The multivariate analysis yielded somewhat different sets of predictors depending on the outcome classifica- tion used. The percent cerebral venous drainage through the jugular veins was identified as an outcome predictor when chief complaint was used for outcome classification. Patients who had an unfavorable outcome had considerably less venous drainage through the jugular veins, which implies increased drainage through the secondary venous channels (e.g., epidural, vertebral, and deep cervical veins). This finding is consistent with previous work that documented an association between type of headache and venous drainage pattern in patients with CM-I, ${ }^{5}$ in which valsalva-related headaches are associated with drainage through the jugular veins and non-valsalva-related headaches are associated with venous drainage through secondary pathways. Altered cerebral venous drainage with increased drainage through secondary veins in the supine posture has been documented in patients with migraine ${ }^{18}$ and idiopathic intracranial hypertension ${ }^{3}$ and in patients who experienced mild traumatic brain injury. ${ }^{25}$ Normally, venous drainage primarily through secondary veins occurs only in an upright posture and drainage primarily through the jugular veins occurs in the supine posture. ${ }^{4}$ It is apparent that patients who had a favorable outcome had the normal pattern of cerebral venous drainage in the supine posture, primarily through the jugular veins. Therefore, the lower percent jugular drainage seen in patients with an unfavorable outcome might reflect an underlying 
pathology separate from CM-I in these patients, leading to the ineffectiveness of treatment directed at CM-I.

\section{Limitations}

This study has several limitations that need to be taken into account when interpreting the data. The primary limitation is the small number of patients in each outcome subcohort, which limits the power of statistical tests, especially for the outcome classification using the chief complaint; only 3 patients fell into the unfavorable-outcome subcohort. In contrast, the CCOS classification had somewhat better power; there were almost equal numbers of patients in each cohort. The disparity between the 2 outcome classifications is a result of the more rigorous criteria of the CCOS, including complete resolution in 2 of 3 domains compared with just the patient's chief compliant. However, regardless of the small number, the study identified outcome predictors, and cord displacement was a consistent predictor across the 2 classification approaches.

Another limitation is the heterogeneity in the clinical presentation. However, all patients in this study met inclusion criteria with the currently accepted definition of CM-I (tonsillar descent $\geq 5 \mathrm{~mm}$ ), and this heterogeneity is representative of the wide anatomical variation between patients with Chiari malformation. Although the findings are clearly limited by the small number of patients and the inherent heterogeneity of the patients with CM-I, the results demonstrate the feasibility of using imaging-based measures, possibly helping to improve treatment outcome for patients with CM-I. The results of this exploratory investigation warrant multicenter studies that could yield a larger number of patients in each of the outcome categories. It should be noted also that this study focused on short-term (3-month) outcomes. Future multicenter studies would allow for longer follow-up periods and assessment of the efficacy of other types of decompressive procedures to further advance and standardize the treatment of CM-I.

\section{Conclusions}

The results of this comparison of morphological and physiological parameters in patients with CM-I with a favorable outcome and in those with an unfavorable outcome after decompressive surgery demonstrate their importance for the diagnosis and treatment of CM-I. Maximal spinal cord displacement emerged as a predictor for outcome of decompressive surgery in patients with CM-I. A favorable outcome was also strongly associated with improved measures of ICC. Patients with reduced cerebral venous drainage through the jugular veins had a less favorable outcome, which may suggest that some of their symptoms were not directly related to the CM-I. These physiological measures demonstrated excellent specificity and sensitivity in predicting surgical outcome. Use of these automated user-independent measures derived from MRI scans tailored for surgical selection of patients with CM-I might lead to a better decompressive surgery success rate.

\section{Acknowledgments}

We thank Lisa Kornse, BSN, RN, for her assistance with patient recruitment. This work was supported by National Institutes of Health Grant R01NS52122.

\section{References}

1. Aliaga L, Hekman KE, Yassari R, Straus D, Luther G, Chen J, et al: A novel scoring system for assessing Chiari malformation type I treatment outcomes. Neurosurgery 70:656665,2012

2. Alperin N, Lee SH: PUBS: pulsatility-based segmentation of lumens conducting non-steady flow. Magn Reson Med 49:934-944, 2003

3. Alperin N, Lee SH, Mazda M, Hushek SG, Roitberg B, Goddwin J, et al: Evidence for the importance of extracranial venous flow in patients with idiopathic intracranial hypertension (IIH). Acta Neurochir Suppl 95:129-132, 2005

4. Alperin N, Lee SH, Sivaramakrishnan A, Hushek SG: Quantifying the effect of posture on intracranial physiology in humans by MRI flow studies. J Magn Reson Imaging 22:591-596, 2005

5. Alperin N, Loftus JR, Oliu CJ, Bagci AM, Lee SH, Ertl-Wagner B, et al: Imaging-based features of headaches in Chiari malformation Type I. Neurosurgery 77:96-103, 2015

6. Alperin N, Loftus JR, Oliu CJ, Bagci AM, Lee SH, ErtlWagner B, et al: Magnetic resonance imaging measures of posterior cranial fossa morphology and cerebrospinal fluid physiology in Chiari malformation type I. Neurosurgery 75:515-522, 2014

7. Alperin N, Sivaramakrishnan A, Lichtor T: Magnetic resonance imaging-based measurements of cerebrospinal fluid and blood flow as indicators of intracranial compliance in patients with Chiari malformation. J Neurosurg 103:46-52, 2005

8. Alperin N, Vikingstad EM, Gomez-Anson B, Levin DN: Hemodynamically independent analysis of cerebrospinal fluid and brain motion observed with dynamic phase contrast MRI. Magn Reson Med 35:741-754, 1996

9. Alperin NJ, Lee SH, Loth F, Raksin PB, Lichtor T: MRIntracranial pressure (ICP): a method to measure intracranial elastance and pressure noninvasively by means of MR imaging: baboon and human study. Radiology 217:877-885, 2000

10. Armonda RA, Citrin CM, Foley KT, Ellenbogen RG: Quantitative cine-mode magnetic resonance imaging of Chiari I malformations: an analysis of cerebrospinal fluid dynamics. Neurosurgery 35:214-224, 1994

11. Badie B, Mendoza D, Batzdorf U: Posterior fossa volume and response to suboccipital decompression in patients with Chiari I malformation. Neurosurgery 37:214-218, 1995

12. Bagci AM, Lee SH, Nagornaya N, Green BA, Alperin N: Automated posterior cranial fossa volumetry by MRI: applications to Chiari malformation type I. AJNR Am J Neuroradiol 34:1758-1763, 2013

13. Dolar MT, Haughton VM, Iskandar BJ, Quigley M: Effect of craniocervical decompression on peak CSF velocities in symptomatic patients with Chiari I malformation. AJNR Am J Neuroradiol 25:142-145, 2004

14. Greenberg JK, Milner E, Yarbrough CK, Lipsey K, Piccirillo JF, Smyth MD, et al: Outcome methods used in clinical studies of Chiari malformation Type I: a systematic review. J Neurosurg 122:262-272, 2015

15. Hofmann E, Warmuth-Metz M, Bendszus M, Solymosi L: Phase-contrast MR imaging of the cervical CSF and spinal cord: volumetric motion analysis in patients with Chiari I malformation. AJNR Am J Neuroradiol 21:151-158, 2000

16. Iskandar BJ, Quigley M, Haughton VM: Foramen magnum cerebrospinal fluid flow characteristics in children with Chiari I malformation before and after craniocervical decompression. J Neurosurg 101 (2 Suppl):169-178, 2004

17. Klekamp J: Surgical treatment of Chiari I malformation- 
analysis of intraoperative findings, complications, and outcome for 371 foramen magnum decompressions. Neurosurgery 71:365-380, 2012

18. Koerte IK, Schankin CJ, Immler S, Lee S, Laubender RP, Grosse C, et al: Altered cerebrovenous drainage in patients with migraine as assessed by phase-contrast magnetic resonance imaging. Invest Radiol 46:434-440, 2011

19. Loth F, Yardimci MA, Alperin N: Hydrodynamic modeling of cerebrospinal fluid motion within the spinal cavity. J Biomech Eng 123:71-79, 2001

20. McGirt MJ, Atiba A, Attenello FJ, Wasserman BA, Datoo G, Gathinji M, et al: Correlation of hindbrain CSF flow and outcome after surgical decompression for Chiari I malformation. Childs Nerv Syst 24:833-840, 2008

21. McGirt MJ, Attenello FJ, Atiba A, Garces-Ambrossi G, Datoo G, Weingart JD, et al: Symptom recurrence after suboccipital decompression for pediatric Chiari I malformation: analysis of 256 consecutive cases. Childs Nerv Syst 24:1333-1339, 2008

22. McGirt MJ, Nimjee SM, Fuchs HE, George TM: Relationship of cine phase-contrast magnetic resonance imaging with outcome after decompression for Chiari I malformations. Neurosurgery 59:140-146, 2006

23. Muehlmann M, Koerte IK, Laubender RP, Steffinger D, Lehner M, Peraud A, et al: Magnetic resonance-based estimation of intracranial pressure correlates with ventriculoperitoneal shunt valve opening pressure setting in children with hydrocephalus. Invest Radiol 48:543-547, 2013

24. Noudel R, Gomis P, Sotoares G, Bazin A, Pierot L, Pruvo JP, et al: Posterior fossa volume increase after surgery for Chiari malformation Type I: a quantitative assessment using magnetic resonance imaging and correlations with the treatment response. J Neurosurg 115:647-658, 2011

25. Pomschar A, Koerte I, Lee S, Laubender RP, Straube A, Heinen F, et al: MRI evidence for altered venous drainage and intracranial compliance in mild traumatic brain injury. PLoS One 8:e55447, 2013

26. Sivaramakrishnan A, Alperin N, Surapaneni S, Lichtor T: Evaluating the effect of decompression surgery on cerebrospinal fluid flow and intracranial compliance in patients with Chiari malformation with magnetic resonance imaging flow studies. Neurosurgery 55:1344-1351, 2004

27. Wang CS, Wang X, Fu CH, Wei LQ, Zhou DQ, Lin JK: Analysis of cerebrospinal fluid flow dynamics and morphology in Chiari I malformation with cine phase-contrast magnetic resonance imaging. Acta Neurochir (Wien) 156:707-713, 2014

28. Yarbrough CK, Greenberg JK, Smyth MD, Leonard JR, Park TS, Limbrick DD Jr: External validation of the Chicago Chiari Outcome Scale. J Neurosurg Pediatr 13:679-684, 2014

\section{Disclosures}

Dr. Alperin is a shareholder in Alperin Noninvasive Diagnostics, Inc., and a holder of the rights for the MRI-ICP patent.

\section{Author Contributions}

Conception and design: Alperin. Acquisition of data: Loftus, Bagci, Lee, Shah, Green. Analysis and interpretation of data: Alperin, Loftus, Bagci, Lee, Oliu. Drafting the article: Alperin. Critically revising the article: Alperin. Reviewed submitted version of manuscript: all authors. Statistical analysis: Loftus, Bagci, Lee. Administrative/technical/material support: all authors. Study supervision: Alperin. Patient recruitment: Green.

\section{Correspondence}

Noam Alperin, Department of Radiology, University of Miami, Ste. 713, Professional Arts Center, 1150 NW 14th St., Miami, FL 33136. email: nalperin@med.miami.edu. 